\title{
ON THE CANONICAL LINE BUNDLE AND NEGATIVE HOLOMORPHIC SECTIONAL CURVATURE
}

\author{
Gordon Heier, Steven S. Y. Lu, And Bun Wong
}

\begin{abstract}
We prove that a smooth complex projective threefold with a Kähler metric of negative holomorphic sectional curvature has ample canonical line bundle. In dimensions greater than three, we prove that, under equal assumptions, the nef dimension of the canonical line bundle is maximal. With certain additional assumptions, ampleness is again obtained. The methods used come from both complex differential geometry and complex algebraic geometry.
\end{abstract}

\section{Introduction}

In complex differential geometry the connection between the holomorphic sectional curvature and the Ricci curvature tensor remains unclear. Many compact Kähler manifolds with negative definite Ricci curvature tensor have no metric with negative holomorphic sectional curvature. The converse is an open problem. To be precise, it is unknown whether there always exists a metric with negative definite Ricci tensor on a compact complex Kähler manifold with negative holomorphic sectional curvature.

In complex dimension two, one can answer this question affirmatively by means of the classification theory of compact complex surfaces. The details of this folklore fact can be found e.g. in [30, Theorem 3.1]. Alternatively, in Section 5.2, we will give a short direct proof for the two-dimensional case that just uses standard algebraic geometry and a theorem of Bishop-Goldberg. This proof also gives a good idea of the general nature of the issues involved. Generalizing it to higher dimension would be very desirable, but seems difficult due to the use of the Riemann-Roch theorem and the lack of information on the Chern numbers.

The main theorem of this paper is the following affirmative answer for algebraic threefolds.

Theorem 1.1. Let $M$ be a smooth projective threefold with a Kähler metric of negative holomorphic sectional curvature. Then its canonical line bundle $K_{M}$ is ample.

Due to the work of Yau [33] (and the Kodaira embedding theorem), it is now well known that the canonical line bundle of a compact Kähler manifold $M$ is ample if and only if there exists (in every Kähler class on $M$ ) a Kähler metric of negative definite Ricci curvature.

Our method is a combination of differential geometric and algebraic geometric machinery. An important step is to eliminate the case when the Kodaira dimension is equal to zero. The paper by Peternell [25] contains a weaker result, but under the more relaxed assumption that $M$ is Brody hyperbolic.

Received by the editors November 24, 2009.

2000 Mathematics Subject Classification. 14C20, 14E05, 32Q45. 
In dimension greater than three, we can establish the conclusion of Theorem 1.1 only with an additional (strong) assumption:

Theorem 1.2. Let $M$ be a projective manifold with a Kähler metric of negative holomorphic sectional curvature. Assume that the essential dimension or the Albanese dimension of $M$ is greater than $\operatorname{dim} M-4$. Then its canonical line bundle $K_{M}$ is ample.

Without additional assumptions, we prove the following weaker positivity statement for $K_{M}$.

Theorem 1.3. Let $M$ be a projective manifold with a Kähler metric with negative holomorphic sectional curvature. Then the nef dimension of $M$ is equal to the dimension of $M$.

\section{Acknowledgements}

We thank the referee for pointing out to us that Theorem 2.3 also follows immediately from a version of Yau's Schwarz lemma [32] found in [34, Theorem 7.23].

\section{Differential geometric background}

Let $M$ be an $n$-dimensional complex manifold with local coordinates $z_{1}, \ldots, z_{n}$. Let

$$
d s^{2}=\sum_{i, j=1}^{n} g_{i \bar{j}} d z_{i} \otimes d \bar{z}_{j}
$$

be a hermitian metric on $M$. The components $R_{i \bar{j} k \bar{l}}$ of the curvature tensor $R$ associated with the hermitian connection are locally given by the formula

$$
R_{i \bar{j} k \bar{l}}=-\frac{\partial^{2} g_{i \bar{j}}}{\partial z_{k} \partial \bar{z}_{l}}+\sum_{p, q=1}^{n} g^{p \bar{q}} \frac{\partial g_{i \bar{p}}}{\partial z_{k}} \frac{\partial g_{q \bar{j}}}{\partial \bar{z}_{l}} .
$$

The Ricci curvature tensor Ric is defined by

$$
\operatorname{Ric}_{k \bar{l}}=\sum_{i, j=1}^{n} g^{i \bar{j}} R_{i \bar{j} k \bar{l}},
$$

and the scalar curvature function $s$ is defined by

$$
s=\sum_{i, j=1}^{n} g^{k \bar{l}} \operatorname{Ric}_{k \bar{l}} .
$$

If $\xi=\sum_{i=1}^{n} \xi_{i} \frac{\partial}{\partial z_{i}}$ is a complex unit vector at $p \in M$, then the holomorphic sectional curvature $H(\xi)$ in the direction of $\xi$ is given by

$$
H(\xi)=2 \sum_{i, j, k, l=1}^{n} R_{i \bar{j} k \bar{l}}(p) \xi_{i} \bar{\xi}_{j} \xi_{k} \bar{\xi}_{l} .
$$

In particular,

$$
H\left(\frac{\partial}{\partial z_{i}}(p)\right)=2 R_{i \bar{i} i \bar{i}}(p)
$$


An important fact about holomorphic sectional curvature is the following. If $M^{\prime}$ is a submanifold of $M$, then the holomorphic sectional curvature of $M^{\prime}$ does not exceed that of $M$. To be precise, if $\xi$ is a unit tangent vector to $M^{\prime}$, then

$$
H^{\prime}(\xi) \leq H(\xi)
$$

where $H^{\prime}$ is the holomorphic sectional curvature associated to the metric on $M^{\prime}$ induced by $d s^{2}$. For a short proof of this inequality see [31, Lemma 1]. Basically, the inequality is an immediate consequence of the Gauss-Codazzi equation.

Compact hermitian manifolds with negative holomorphic sectional curvature enjoy the remarkable property that they do not accommodate an entire complex curve. A generalized Schwarz lemma due to Ahlfors implies the following useful result.

Theorem 2.1. Let $M$ be a hermitian manifold with holomorphic sectional curvature bounded above by a negative constant. Then there exists no non-constant holomorphic map from the complex plane into $M$ (i.e., $M$ is Brody hyperbolic).

In particular, on a compact hermitian manifold with negative holomorphic sectional curvature, there exist no (birational images of) rational or elliptic curves.

Although it is in general unknown if one can find a metric with negative definite Ricci curvature on a compact hermitian manifold with negative holomorphic sectional curvature, we have the following pointwise result due to Berger [3].

Theorem 2.2. The scalar curvature of a Kähler metric with negative holomorphic sectional curvature is also negative.

We recall that a hermitian metric $\sum_{i, j=1}^{n} g_{i \bar{j}} d z_{i} \otimes d \bar{z}_{j}$ is Kähler if and only if locally there exists a real-valued function $f$ such that $g_{i \bar{j}}=\frac{\partial^{2} f}{\partial z_{i} \partial \bar{z}_{j}}$.

Berger's theorem is proven using a pointwise formula expressing the scalar curvature at a point in terms of the average holomorphic sectional curvature on the unit sphere in the tangent space at that point. It does not seem to be a strong enough statement to immediately yield results concerning the Ricci curvature. Nevertheless, it allows us to prove the following fact, which will play a crucial role in our argument to rule out the Calabi-Yau case. Since we were unable to find a proof in the literature, we provide one here.

Theorem 2.3. There exists no Kähler metric with negative holomorphic sectional curvature on a compact Kähler manifold $M$ with zero first real Chern class.

Proof. We will make use of the following formula, which is a special case of a formula due to Chern. Let

$$
u=\frac{v_{2}}{v_{1}}
$$

where $v_{1}, v_{2}$ are the respective volume forms associated to two metrics $d s_{1}^{2}, d s_{2}^{2}$ on $M$. Then $[8$, Formula (3)] reads

$$
\frac{1}{2} \Delta \ln u=s-\operatorname{Tr}(\mathrm{Ric}),
$$

where $\Delta$ is the Laplacian operator associated with $d s_{1}^{2}, \operatorname{Tr}$ denotes the trace with respect to $d s_{1}^{2}, s$ is the scalar curvature of $d s_{1}^{2}$, and Ric is the Ricci curvature tensor of $d s_{2}^{2}$. 
Assume $d s_{1}^{2}$ is a Kähler metric with negative holomorphic sectional curvature. By Theorem 2.2, the scalar curvature of $d s_{1}^{2}$ is negative. Moreover, Yau's Theorem [33] says that there exists a Kähler metric $d s_{2}^{2}$ with vanishing Ricci curvature tensor.

Let $p$ be the point at which $\ln u$ achieves its minimum. By the second derivative test, $(\Delta \ln u)(p) \geq 0$. On the other hand, since Ric $\equiv 0$, it follows from Chern's formula that

Contradiction.

$$
\frac{1}{2}(\Delta \ln u)(p)=s(p)<0 .
$$

Remark 2.4. Since we will see in Step 1 of the proof of Theorem 1.1 that a compact manifold $M$ with a Kähler metric of negative holomorphic sectional curvature has nef $K_{M}$, we note that Theorem 2.3 can be rephrased as saying that a compact manifold $M$ with a Kähler metric of negative holomorphic sectional curvature has strictly positive numerical dimension $\nu(M)$. Recall that the numerical dimension $\nu(M)$ is $\max \left\{k \in\{0,1, \ldots, \operatorname{dim} M\}:\left(c_{1}^{\mathbb{R}}(M)\right)^{k} \not \equiv 0\right\}$ (when $K_{M}$ is nef).

We conclude this section with the following corollary to Theorem 2.3.

Corollary 2.5. Let $M$ be a projective manifold of Picard number one with a Kähler metric of negative holomorphic sectional curvature. Then $K_{M}$ is ample.

Proof. We will see in Step 1 of the proof of Theorem 1.1 that $K_{M}$ is nef. Since $K_{M}$ is not numerically trivial by Theorem 2.3 , its numerical class must be a positive multiple of the numerical class of an arbitrarily chosen ample divisor. According to the Nakai-Moishezon-Kleiman criterion [19, Theorem 1.2.23], this shows that $K_{M}$ is ample.

\section{Proof of Theorem 1.1}

The major task is to deal with the case when the Kodaira dimension of $M$, denoted by $\operatorname{kod}(M)$, is equal to zero. We recall the definition.

Definition 3.1. Let $M$ be a projective manifold and $K_{M}$ its canonical line bundle. If $H^{0}\left(M, m K_{M}\right)=0$ for all $m>0$, then $\operatorname{kod}(M)=-\infty$. If this is not the case, then we take $\operatorname{kod}(M)$ to be the maximal dimension of the images of $M$ under the rational maps furnished by non-empty linear systems $\left|m K_{M}\right|(m>0)$.

We break our arguments into five steps as follows.

Step 1. Recall that a line bundle $L$ on a projective manifold is nef if $L . C \geq 0$ for all curves $C$ on $M$. If the canonical line bundle $K_{M}$ of $M$ is not nef, then there exists a curve $C$ such that $K_{M} . C<0$. Then Mori's theory ([23], [18]) yields that there exists a rational curve on $M$ (regardless of $\operatorname{dim} M$ ). This is disallowed by Theorem 2.1. Hence we can assume that $K_{M}$ is nef from now on.

Step 2. The following is a theorem of Miyaoka [22, Theorem 1.1] that yields the existence of some pluricanonical sections.

Theorem 3.2. Let $M$ be a smooth projective threefold with nef canonical line bundle. Then $H^{0}\left(M, m K_{M}\right)$ is non-zero for some $m>0$. In other words, $\operatorname{kod}(M) \geq 0$.

Step 3. If $1 \leq \operatorname{kod}(M) \leq 2$, we consider the Kodaira-Iitaka map $\pi: \tilde{M} \rightarrow Y$ (see [12]). This is a surjective holomorphic map of projective manifolds such that 
(i) $\tilde{M}$ is birational to $M$,

(ii) $\operatorname{dim} Y=\operatorname{kod}(M)$,

(iii) a general fiber $F$ of $\pi$ is irreducible and smooth with $\operatorname{kod}(F)=0$.

Firstly, if $F$ is a smooth curve with $\operatorname{kod}(F)=0$, then $F$ is an elliptic curve. Secondly, $F$ may be a smooth surface with $\operatorname{kod}(F)=0$. From the Enriques-Kodaira classification we know that the minimal model of $F$ can only be a hyperelliptic surface, a torus, a projective K3 surface or an Enriques surface. For the following reasons, all these surfaces admit a one parameter family of entire curves. This property then holds also for $F$, because it is preserved under birational maps of surfaces.

A hyperelliptic surface is a locally trivial fibration whose typical fiber is an elliptic curve.

A torus is covered by the images of non-constant entire curves simply because its universal cover is $\mathbb{C}^{2}$.

It is a theorem of Bogomolov-Mumford [24, Appendix] that a projective K3 surface contains a one parameter family of singular elliptic curves.

Finally, the universal cover of any Enriques surface is a projective K3 surface.

The birational map $\sigma: \tilde{M} \rightarrow M$ is a holomorphic surjection. The restriction of $\sigma$ to a general fiber $F$ of $\pi$, both in the cases of $F$ being a curve and $F$ being a surface, is generically injective. Thus, many entire curves are mapped non-trivially into $M$. This is a contradiction to the Brody hyperbolicity of $M$.

Step 4. If $\operatorname{kod}(M)=3$, then $K_{M}$ is big (and nef). The following proposition is a consequence of [15] or [16] or [9, Exercise 10.1] and proves Theorem 1.1 in this case.

Proposition 3.3. Let $M$ be a complex projective Brody hyperbolic manifold with $K_{M}$ big. Then $K_{M}$ is ample.

Step 5. The final and most important case to rule out is $\operatorname{kod}(M)=0$. Once we have proven that all such algebraic threefolds cannot admit a Kähler metric with negative holomorphic sectional curvature, the proof of Theorem 1.1 is complete.

Since $\operatorname{kod}(M)=0$, there exists $m>0$ with $\operatorname{dim} H^{0}\left(M, m K_{M}\right)=1$. Let $\sigma \in$ $H^{0}\left(M, m K_{M}\right) \backslash\{0\}$. If $\operatorname{div}(\sigma)=\emptyset$, then $m K_{M}$ is the trivial line bundle. In particular, its first Chern class is zero. Consequently, the first real Chern class of $K_{M}$ is zero. This is impossible due to Theorem 2.3.

We finally have to treat the situation when $\operatorname{div}(\sigma)=\sum_{i=1}^{T} k_{i} D_{i}$, where $T \geq 1$, $k_{i} \geq 1$ and $D_{i}$ a prime divisor. In order to do so, we need the following result due to Wilson [29].

Theorem 3.4. With the above notation, for a given index $i$, one of the following is true.

(i) $D_{i}$ is birational to a ruled surface.

(ii) $D_{i}$ has at worst rational double point singularities. Its desingularization $\hat{D}_{i}$ satisfies $\operatorname{kod}\left(\hat{D}_{i}\right)=0$.

In our situation, the case (i) cannot occur, since it would constitute an obvious contradiction to the Brody hyperbolicity. As for case (ii), we saw above that $\hat{D}_{i}$ and $D_{i}$ cannot be Brody hyperbolic. Contradiction. 


\section{Proofs of Theorems 1.2 and 1.3}

4.1. The case of the essential dimension. The essential dimension is based on Campana's construction of the core of a projective manifold in [7] (see also [21] for an alternative but equivalent construction). We think of the core as described in $[7$, Theorem 3.3], namely as an almost holomorphic fibration $c: M \rightarrow C(M)$, where $C(M)$ is an orbifold which is of general type in a suitable sense. Moreover, a general fiber $F$ of $c$ is special. We refer to [7] for the exact definition of special. It suffices for us to note that, in particular, special projective manifolds are not of maximal Kodaira dimension. The dimension of $C(M)$ is called the essential dimension of $M$.

The proof of Theorem 1.2 for the case of the essential dimension is basically immediate from Theorem 1.1. We assume that the Kodaira dimension of $M$ is not maximal and derive a contradiction. If the essential dimension is greater than $\operatorname{dim} M-4$, then the dimension of $F$ is 1,2, or 3. By the properties of $c, F$ is not of general type. On the other hand, due to the curvature decreasing property described in Section $2, F$ itself is a projective manifold with a Kähler metric of negative holomorphic sectional curvature. The case of $\operatorname{dim} F=1$ being trivial, we can conclude the proof by noting that our results from Section $5.2(\operatorname{dim} F=2)$ and Theorem $1.1(\operatorname{dim} F=3)$ show that $K_{F}$ is ample. Contradiction.

4.2. The case of the Albanese dimension. Let $a: M \rightarrow T$ be the Albanese mapping of $M$ to its Albanese torus $T$ (see [11]). Then the Albanese dimension of $M$ is defined to be $\operatorname{dim} a(M)$. If the Albanese dimension of $M$ is greater than $\operatorname{dim} M-4$, then the map $a: M \rightarrow a(M)$ is a holomorphic map such that an irreducible component $F$ of a general fiber has dimension at most 3. W.l.o.g., we assume that the dimension of $F$ is equal to 3 . By the same token as above, $\operatorname{kod}(F)=\operatorname{dim} F$. Again, we assume that the Kodaira dimension of $M$ is not maximal and derive a contradiction.

Let $\pi: \tilde{M} \rightarrow Z$ be a holomorphic version of the Kodaira-Iitaka map of $M$ and $\sigma: \tilde{M} \rightarrow M$ the pertaining modification of $M$. We denote by $\tilde{F}$ the strict transform of $F$ under $\sigma$, which also has maximal Kodaira dimension.

The restriction of $\pi$ to $\tilde{F}$ gives a holomorphic map $\pi: \tilde{F} \rightarrow \pi(\tilde{F})$. If we denote an irreducible component of a general fiber of this map by $S$, then the Easy Addition Formula (see [13]) yields

$$
3=\operatorname{kod}(\tilde{F}) \leq \operatorname{kod}(S)+\operatorname{dim} \pi(\tilde{F}) .
$$

Since clearly $3=\operatorname{dim} \tilde{F}=\operatorname{dim} S+\operatorname{dim} \pi(\tilde{F})$, we see that $S$ is of maximal Kodaira dimension.

On the other hand, let $G$ be the fiber of $\pi: \tilde{M} \rightarrow Z$ such that $S$ is a component of $G \cap \tilde{F}$. By the standard properties of the Kodaira-Iitaka map, when $F$ and $S$ are appropriately chosen, $G$ is a projective manifold with $\operatorname{kod}(G)=0$. By the subadditivity theorem of Kollár [17] and Viehweg [28] for the case of fibers of general type, we have

$$
0=\operatorname{kod}(G) \geq \operatorname{kod}(S)+\operatorname{kod}(a(\sigma(G))) .
$$

Note that $a(\sigma(G))$ may be singular, so we define its Kodaira dimension to be that of a desingularization. By [27, Theorem 10.9], we know that $\operatorname{kod}(a(\sigma(G)))$ is at least 0 . Hence, since $S$ is of maximal Kodaira dimension, $0=\operatorname{kod}(S)=\operatorname{kod}(a \circ \sigma(G))$. Consequently, $S$ is a point. 
The fact that $S$ is a point implies that $a \circ \sigma: G \rightarrow a(\sigma(G))$ is a generically finite holomorphic map. If we denote by $b$ the Albanese map of $G$, then Kawamata's [14, Theorem 1] yields that $b$ is a fibration. By the universal property of $b$, the holomorphic map $a \circ \sigma: G \rightarrow a(\sigma(G)) \subset T$ factors through $b$. However, this clearly means that the general fibers of $b$ are zero-dimensional. Consequently, $G$ is birational to its Albanese torus, whence $G$ and $\sigma(G)$ are not hyperbolic. Thus, $\tilde{M}$ and $M$ are not hyperbolic. Contradiction. This concludes the proof of Theorem 1.2.

4.3. The nef dimension: Proof of Theorem 1.3. Since it is very difficult to produce a section of $m K_{M}$ solely based on the fact that $K_{M}$ is nef, it is tempting to consider the nef dimension and nef reduction map introduced by Tsuji ([26], see also $[5],[6],[2])$.

Let $M$ be a projective manifold with nef canonical line bundle. We define two distinct points $p$ and $q$ of $M$ to be equivalent ( $p \sim q$ ") if and only if there exists a connected (not necessarily irreducible) curve $C$ containing both $p$ and $q$ such that $K_{M} . C=0$. According to [6], there is an almost holomorphic rational map $f: M \rightarrow$ $Y$ with connected fibers to a normal projective variety $Y$ such that for generic points $p, q$ in $M, p \sim q$ if and only if $f(p)=f(q)$. The precise statement from [2] on the nef reduction map is the following.

Theorem 4.1. Let $L$ be a nef line bundle on a normal projective variety $M$. Then there exists an almost holomorphic dominant rational map $f: M \rightarrow Y$ with connected fibers, called a "reduction map," such that

(i) $L$ is numerically trivial on all compact fibers $F$ of $f$ with $\operatorname{dim} F=\operatorname{dim} M-$ $\operatorname{dim} Y$, and

(ii) for every general point $x \in M$ and every irreducible curve $C$ passing through $x$ with $\operatorname{dim} f(C)>0$, we have L.C $>0$.

The map $f$ is unique up to birational equivalence of $Y$.

When we apply the above theorem with $L=K_{M}$, we call $n(M):=\operatorname{dim} Y$ the nef dimension of $M$. The advantage of dealing with the nef dimension is that its definition does not appeal to the existence of any non-trivial pluricanonical sections.

We conclude this section with the

Proof of Theorem 1.3. Assume that $n(M)<\operatorname{dim} M$, and let $k:=\operatorname{dim} M-n(M)>0$. Then the general fiber $F$ of the above reduction map $f$ is a projective manifold of dimension $k$ such that $K_{M} . C=0$ for all curves $C$ in $F$. By the adjunction formula, $\left.K_{M}\right|_{F}=K_{F}$, and consequently $K_{F}$ is numerically trivial. However, this means that the first real Chern class of $F$ is trivial (see [19, Remark 1.1.20]). Due to the curvature decreasing property and Theorem 2.3, we get a contradiction. (Note that if $n(M)=0$, we work with $F=M$.)

\section{Alternative approaches}

5.1. Use of the abundance conjecture. The abundance conjecture in algebraic geometry says that on a projective manifold $M$ with nef canonical line bundle $K_{M}$, there exists an integer $m>0$ such that $m K_{M}$ is base point free. If this conjecture is true, then our Theorem 1.1 would be true in arbitrary dimension. The proof would go as follows. 
Consider the holomorphic map $\pi: M \rightarrow Y$ furnished by the base point free linear system $\left|m K_{M}\right|$. If $0<\operatorname{kod}(M)<\operatorname{dim} M$, the general fiber of $\pi$ is a projective manifold $F$ with $0<\operatorname{dim} F<\operatorname{dim} M$ and $\operatorname{kod}(F)=0$. Moreover, due to the curvature decreasing property of submanifolds discussed in Section 2, the induced Kähler metric on $F$ has negative holomorphic sectional curvature. This can be ruled out by induction.

In the case $\operatorname{kod}(M)=0$, we have $\operatorname{dim} H^{0}\left(M, m K_{M}\right) \in\{0,1\}$ for all $m>0$. When we take $m$ to be such that $m K_{M}$ is base point free, then $\operatorname{dim} H^{0}\left(M, m K_{M}\right)=1$, and $m K_{M}$ is clearly trivial. Consequently, $K_{M}$ has trivial first real Chern class, and one again obtains a contradiction due to Theorem 2.3.

Finally, in the case $\operatorname{kod}(M)=\operatorname{dim} M$, one shows that $K_{M}$ is ample as we $\operatorname{did}$ in Step 4 of the proof of Theorem 1.1 .

Unfortunately, the abundance conjecture is open in dimension at least four, although some special cases are known, such as the case of irregular fourfolds [10]. Moreover, using the abundance conjecture seems to be overkill in any case, since a more specialized argument making better use of the negative curvature assumption seems much more appropriate. In this spirit, we show in Section 5.2 how to use the negative curvature assumption directly to prove our main theorem in two dimensions without any sort of fibration argument.

5.2. The two-dimensional case. Let $S$ be a complex projective surface with a Kähler metric of negative holomorphic sectional curvature. In this subsection, we show that $K_{S}$ is ample using only standard algebraic geometry and a theorem from $[4]$.

We have already noted that $K_{S}$ is nef. However, more is true:

Lemma 5.1. Let $S$ be as above. Then $K_{S}$ is strictly nef, i.e., $K_{S} . C>0$ for all curves $C$ in $S$.

Proof. Assume that there is a curve $C$ with $K_{S} \cdot C \leq 0$. Since $K_{S}$ is nef, this means $K_{S} . C=0$. Because of the Brody hyperbolicity, the arithmetic genus $g(C)$ is at least 2 , and thus by the adjunction formula

$$
2 \leq 2 g(C)-2=K_{S} \cdot C+C^{2}=C^{2} .
$$

The Hodge Index Theorem [1] tells us that $C^{2}>0$ implies that for any class $[\omega] \in$ $H_{\mathbb{R}}^{1,1}(S)$ such that $\int_{C} \omega=0$, we have $\int_{M} \omega \wedge \omega \leq 0$. Moreover, $\int_{M} \omega \wedge \omega=0$ if and only if $[\omega]=0$. However, since $K_{S}$ is nef, we know that $K_{S}^{2}=\int_{M} c_{1}^{\mathbb{R}}\left(K_{S}\right) \wedge c_{1}^{\mathbb{R}}\left(K_{S}\right) \geq 0([19$, Theorem 1.4.9. (Kleiman's theorem)]). According to the above with $[\omega]=c_{1}^{\mathbb{R}}\left(K_{S}\right)$, this is only possible if $c_{1}^{\mathbb{R}}\left(K_{S}\right)=0$. This is a contradiction due to Theorem 2.3.

According to the Nakai-Moishezon-Kleiman criterion [19, Theorem 1.2.23], $K_{S}$ is ample if we can show that $K_{S}^{2}>0$. We formulate this as a lemma.

Lemma 5.2. Let $S$ be as above. Then $K_{S}^{2}>0$.

Proof. Since we already know $K_{S}^{2}$ to be non-negative, we just need to assume $K_{S}^{2}=0$ and derive a contradiction.

Since $K_{S}$ is strictly nef according to Lemma 5.1, there exists a very ample divisor $H$ such that $K_{S} \cdot H>0$. 
We now write down the Riemann-Roch theorem for $2 K_{S}$ (see [11, p.472]):

$$
h^{0}\left(2 K_{S}\right)-h^{1}\left(2 K_{S}\right)+h^{2}\left(2 K_{S}\right)=\frac{1}{12}\left(K_{S}^{2}+c_{2}(S)\right)+\frac{1}{2}\left(\left(2 K_{S}\right)^{2}-2 K_{S}^{2}\right) .
$$

Note that by Serre duality, $h^{2}\left(2 K_{S}\right)=h^{0}\left(-K_{S}\right)$. If this number is different from zero, then there is an effective divisor $D$ in the linear system $\left|-K_{S}\right|$ such that $D . H \geq 0$. This is a contradiction to $D . H=-K_{S} \cdot H<0$. Consequently, equation (1) can be simplified to

$$
h^{0}\left(2 K_{S}\right) \geq \frac{1}{12} c_{2}(S) .
$$

Due to the Gauss-Bonnet theorem, $c_{2}(S)$ is just the topological Euler characteristic of $S$. However, [4, Theorem 1.3] says that any compact Kähler surface of definite (positive or negative) holomorphic sectional curvature has positive Euler characteristic. Thus we can conclude that $h^{0}\left(2 K_{S}\right)>0$.

Finally, to derive the contradiction, let $C \in\left|2 K_{S}\right|$ be an effective divisor. Since $K_{S}$ is strictly nef by Lemma 5.1 , we have

$$
0<C . K_{S}=2 K_{S} \cdot K_{S}=2 K_{S}^{2}=2 \cdot 0=0 .
$$

\section{References}

[1] W. Barth, C. Peters, and A. Van de Ven, Compact complex surfaces, Vol. 4 of Ergebnisse der Mathematik und ihrer Grenzgebiete (3) [Results in Mathematics and Related Areas (3)], Springer-Verlag, Berlin (1984), ISBN 3-540-12172-2.

[2] T. Bauer, F. Campana, T. Eckl, S. Kebekus, T. Peternell, S. Rams, T. Szemberg, and L. Wotzlaw, A reduction map for nef line bundles, in Complex geometry (Göttingen, 2000), 27-36, Springer, Berlin (2002).

[3] M. Berger, Sur les variétés d'Einstein compactes, in Comptes Rendus de la IIIe Réunion du Groupement des Mathématiciens d'Expression Latine (Namur, 1965), 35-55, Librairie Universitaire, Louvain (1966).

[4] R. L. Bishop and S. I. Goldberg, Some implications of the generalized Gauss-Bonnet theorem, Trans. Amer. Math. Soc. 112 (1964) 508-535.

[5] F. Campana, Coréduction algébrique d'un espace analytique faiblement kählérien compact, Invent. Math. 63 (1981), no. 2, 187-223.

[6] — , Remarques sur le revêtement universel des variétés kählériennes compactes, Bull. Soc. Math. France 122 (1994), no. 2, 255-284.

[7] - Orbifolds, special varieties and classification theory, Ann. Inst. Fourier (Grenoble) $\mathbf{5 4}$ (2004), no. 3, 499-630.

[8] S. Chern, On holomorphic mappings of hermitian manifolds of the same dimension., in Entire Functions and Related Parts of Analysis (Proc. Sympos. Pure Math., La Jolla, Calif., 1966), 157-170, Amer. Math. Soc., Providence, R.I. (1968).

[9] O. Debarre, Lecture Notes, Summer School: Geometry of complex projective varieties and the minimal model program, Grenoble (2007).

[10] O. Fujino, Finite generation of the log canonical ring in dimension four, arXiv:math.AG/ 0803.1691 (2008)

[11] P. Griffiths and J. Harris, Principles of algebraic geometry, Wiley Classics Library, John Wiley \& Sons Inc., New York (1994), ISBN 0-471-05059-8. Reprint of the 1978 original.

[12] S. Iitaka, On D-dimensions of algebraic varieties, Proc. Japan Acad. 46 (1970) 487-489.

[13] - Algebraic geometry, Vol. 76 of Graduate Texts in Mathematics, Springer-Verlag, New York (1982), ISBN 0-387-90546-4. An introduction to birational geometry of algebraic varieties, North-Holland Mathematical Library, 24. 
[14] Y. Kawamata, Characterization of abelian varieties, Compositio Math. 43 (1981), no. 2, 253276.

[15] - Minimal models and the Kodaira dimension of algebraic fiber spaces, J. Reine Angew. Math. 363 (1985) 1-46.

[16] - On the length of an extremal rational curve, Invent. Math. 105 (1991), no. 3, 609-611.

[17] J. Kollár, Subadditivity of the Kodaira dimension: fibers of general type, in Algebraic geometry, Sendai, 1985, Vol. 10 of Adv. Stud. Pure Math., 361-398, North-Holland, Amsterdam (1987).

[18] J. Kollár and S. Mori, Birational geometry of algebraic varieties, Vol. 134 of Cambridge Tracts in Mathematics, Cambridge University Press, Cambridge (1998), ISBN 0-521-63277-3. With the collaboration of C. H. Clemens and A. Corti, Translated from the 1998 Japanese original.

[19] R. Lazarsfeld, Positivity in algebraic geometry. I, Vol. 48 of Ergebnisse der Mathematik und ihrer Grenzgebiete. 3. Folge. A Series of Modern Surveys in Mathematics [Results in Mathematics and Related Areas. 3rd Series. A Series of Modern Surveys in Mathematics], Springer-Verlag, Berlin (2004), ISBN 3-540-22533-1. Classical setting: line bundles and linear series.

[20] S. S. Y. Lu, The Kobayashi pseudometric on algebraic manifold and a canonical fibration, arXiv:math.AG/0206170 (2002).

[21] — A refinded Kodaira dimension and its canonical fibration, arXiv:math.AG/0211029 (2002).

[22] Y. Miyaoka, On the Kodaira dimension of minimal threefolds, Math. Ann. 281 (1988), no. 2, 325-332.

[23] S. Mori, Threefolds whose canonical bundles are not numerically effective, Ann. of Math. (2) 116 (1982), no. 1, 133-176.

[24] S. Mori and S. Mukai, The uniruledness of the moduli space of curves of genus 11, in Algebraic geometry (Tokyo/Kyoto, 1982), Vol. 1016 of Lecture Notes in Math., 334-353, Springer, Berlin (1983).

[25] T. Peternell, Calabi-Yau manifolds and a conjecture of Kobayashi, Math. Z. 207 (1991), no. 2, 305-318.

[26] H. Tsuji, Numerically trivial fibrations, arXiv:math.AG/0001023 (2000).

[27] K. Ueno, Classification theory of algebraic varieties and compact complex spaces, Lecture Notes in Mathematics, Vol. 439, Springer-Verlag, Berlin (1975). Notes written in collaboration with P. Cherenack.

[28] E. Viehweg, Weak positivity and the stability of certain Hilbert points, Invent. Math. 96 (1989), no. 3, 639-667.

[29] P. M. H. Wilson, The components of $m K_{V}$ for threefolds with $\kappa(V)=0$, Math. Proc. Cambridge Philos. Soc. 97 (1985), no. 3, 437-443.

[30] B. Wong, The uniformization of compact Kähler surfaces of negative curvature, J. Differential Geom. 16 (1981), no. 3, 407-420 (1982).

[31] H. Wu, A remark on holomorphic sectional curvature, Indiana Univ. Math. J. $22(1972 / 73)$ 1103-1108.

[32] S. T. Yau, A general Schwarz lemma for Kähler manifolds, Amer. J. Math. 100 (1978), no. 1, 197-203.

[33] - On the Ricci curvature of a compact Kähler manifold and the complex Monge-Ampère equation. I, Comm. Pure Appl. Math. 31 (1978), no. 3, 339-411.

[34] F. Zheng, Complex differential geometry, Vol. 18 of AMS/IP Studies in Advanced Mathematics, American Mathematical Society, Providence, RI (2000), ISBN 0-8218-2163-6.

Department of Mathematics, University of Houston, 4800 Calhoun Road, Houston, TX 77204, USA

E-mail address: heier@math.uh.edu

Départment de Mathématiques, Université Du QéBec À Montréal, C.P. 8888, Succursale Centre-Ville, Montréal, QC H3C 3P8, Canada

E-mail address: lu.steven@uqam.ca

Department of Mathematics, UC Riverside, 900 University Avenue, Riverside, CA 92521, USA

E-mail address: wong@math.ucr.edu 